\title{
EL FIN (MOMENTÁNEO) DEL BIPARTIDISMO EN ESPAÑA: ANÁLISIS DE LOS RESULTADOS ELECTORALES DE 2015 Y 2016
}

\author{
The (Momentary) End of Two-Party System in Spain: \\ Analysis of the Electoral Results of 2015 and 2016
}

\author{
ÓSCAR SÁNCHEZ MUÑOZ \\ Universidad de Valladolid \\ sanchez@der.uva.es
}

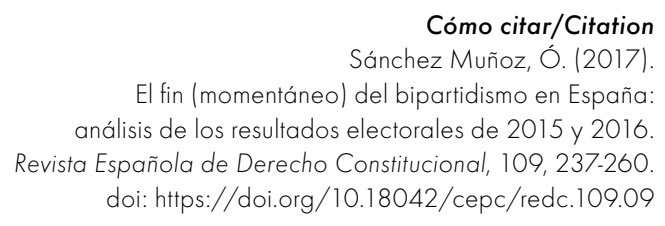

Resumen

Los resultados de las elecciones generales de 2015 y 2016 en España han tenido como resultado una reconfiguración del sistema de partidos. Se ha pasado del bipartidismo imperfecto a un multipartidismo fragmentado. Estos resultados entran en aparente contradicción con la hasta ahora probada capacidad del sistema electoral del Congreso de los Diputados para fabricar mayorías parlamentarias. En este artículo se analiza el impacto real del sistema electoral sobre los resultados de 2015 y 2016 y su posible influencia en la evolución futura del sistema de partidos.

\section{Palabras clave}

Elecciones; sistema electoral; sistema de partidos; representación proporcional. 


\begin{abstract}
The results of the general elections of 2015 and 2016 in Spain led to a reconfiguration of the party system, moving from an imperfect two-party system to a fragmented multi-party system. These results are in apparent contradiction with the hitherto proven capacity of the electoral system of the Congress of Deputies to manufacture parliamentary majorities. The article analyses the real impact of the electoral system on the results of 2015 and 2016 and its possible influence on the future evolution of the party system.
\end{abstract}

\title{
Keywords
}

Elections; electoral system; party system; proportional representation. 
EL FIN (MOMENTÁNEO) DEL BIPARTIDISMO EN ESPAÑA: ANÁLISIS DE LOS RESULTADOS... 239

\section{SUMARIO}

I. INTRODUCCIÓN. II. LOS EFECTOS DEL SISTEMA ELECTORAL Y SU IMPACTO EN LOS RESULTADOS DE 2015 Y 2016: 1. Un sistema electoral pensado para fabricar mayorías y, especialmente, mayorías conservadoras. 2. ¿Qué ha pasado en 2015 y 2016 ? III. LA POSIBLE INFLUENCIA DEL SISTEMA ELECTORAL EN LA EVOLUCIÓN FUTURA DEL SISTEMA DE PARTIDOS... Y VICEVERSA. IV. UNA REFLEXIÓN FINAL: ¿̇SIMPLE REALINEAMIENTO O TRANSFORMACIÓN PROFUNDA DEL SISTEMA DE PARTIDOS? BIBLIOGRAFÍA.

\section{INTRODUCCIÓN}

Como consecuencia de la crisis económica iniciada en 2008, muchos estados europeos se han visto sometidos a cambios políticos de diverso calado que resultan aún difíciles de evaluar. El rechazo a las políticas coyunturales desarrolladas por los Gobiernos ha confluido con un fenómeno estructural de malestar o desafección respecto a las instituciones democráticas y a los partidos políticos que se viene manifestando en todas las democracia contemporáneas desde hace un par de décadas. España, especialmente tocada por la crisis, no ha sido ajena a estas turbulencias políticas. El fenómeno del 15-M y las elecciones de 2011, que se saldaron con la mayoría absoluta del Partido Popular (PP) y el batacazo electoral del Partido Socialista Obrero Español (PSOE), ya mostraron los primeros síntomas de los cambios que se avecinaban, los cuales se hicieron aún más patentes en las elecciones europeas de 2014 —con la aparición en la escena política de Podemos-y en las municipales y autonómicas de mayo de 2015.

Las elecciones legislativas de 20 de diciembre de 2015 y de 26 de junio de 2016 han tenido como resultado una reconfiguración del sistema de partidos que ha predominado en nuestro país desde las primeras elecciones democráticas celebradas el 15 de junio de 1977.

De aquel primer proceso electoral de la democracia surgió un bipartidismo imperfecto que se ha mantenido en sus rasgos esenciales hasta 2015: un sistema articulado por la confrontación entre dos fuerzas políticas dominantes separadas por una línea divisoria (cleavage) fundamentalmente ideológica. Tras un período inicial de consolidación de ambos polos no exento de complejidad, pues «la formación de un sistema de partidos después de cuarenta años de régimen dictatorial fue uno de los principales problemas políticos de la Transición, directamente asociado con la cuestión de la gobernabilidad en los primeros 
años de la nueva democracia española» (Maravall y Santamaría, 1989: 218), los papeles protagonistas de este sistema fueron asumidos por el PSOE y el PP, debiéndose notar que el período de consolidación fue más prolongado y accidentado para el centro-derecha, con la crisis e implosión de la Unión de Centro Democrático (UCD) y la realineación de las fuerzas conservadoras en torno a Alianza Popular (AP), refundada más tarde (en 1989) como PP.

Fue un bipartidismo imperfecto en un doble sentido. De una parte, porque ninguno de los dos partidos logró aglutinar el $100 \%$ de su respectivo espacio ideológico y, de otra parte, porque a la línea divisoria fundamental que articulaba el sistema, que era la escisión ideológica izquierda-derecha, se le sumaba también una línea divisoria territorial, representada por la oposición entre partidos nacionales y partidos nacionalistas. De la combinación de ambas divisiones, y con la ayuda de un sistema electoral del que hablaremos más adelante, surgía un tablero sin duda complejo, pero en el que predominaba siempre la bipolaridad.

La suma de los votos de los dos partidos más votados en elecciones generales alcanzó el 63,76 \% de los votos válidos en 1977, el 65,24\% en 1979, el $74,47 \%$ en 1982 , el $70,03 \%$ en 1986 , el $65,39 \%$ en 1989 , el $73,54 \%$ en 1993, el 76,42\% en 1996, el 78,68 \% en 2000, el 80,3\% en 2004, el 83,81\% en 2008 (máximo histórico) y el 73,39 \% en 2011. Son cifras que resisten perfectamente la comparación con algunos de los sistemas más marcadamente bipartidistas de Europa. Por ejemplo, en el Reino Unido, durante el mismo período de tiempo, la suma de los votos obtenidos por los dos primeros partidos en las elecciones legislativas osciló entre el 65,4\% en 2010 (primer Gobierno de Cameron, máxima cota de los liberal-demócratas) y el 80,8 \% en 1979 (primera victoria de Thatcher). En Alemania, la suma de los votos de los dos grandes partidos ciertamente superó el $90 \%$ en los años setenta (en la época dorada de Willy Brandt), pero luego fue descendiendo — sobre todo tras la reunificación-y en los años noventa se situó en torno al 77 \% y bajó por debajo del $70 \%$ en la primera década de este siglo, hasta alcanzar la cota más baja, de momento, en 2009 con el 56,8 \%.

Otra de las características del modelo bipartidista que predominó en España hasta 2015 era que las dos fuerzas predominantes, en tanto opciones de Gobierno nacional, se presentaban ante el electorado como mutuamente excluyentes. Es decir, si había un Gobierno del PSOE, ello excluía al PP y viceversa. No había ninguna posibilidad de colaboración. En ese sentido, podemos decir que no era solo un sistema bipartidista, sino también un sistema caracterizado por una fuerte polarización, en algunos momentos extrema.

Las elecciones generales de diciembre de 2015 rompen completamente con este bipartidismo imperfecto. La suma de los votos de los dos principales 
partidos (PP y PSOE) se queda en un 50,71\% de los votos válidos. En las elecciones de junio de 2016, ambos llegan al 55,64\% debido a la remontada del PP, importante en número absoluto de votos recibidos respecto a la convocatoria anterior (+704 271 votos), pero aún más en cuanto al porcentaje de voto, habida cuenta de la elevada abstención que se produjo (33,52 \%), la cual afectó fundamentalmente al voto de izquierda.

¿Realmente nos encontramos ante el fin del bipartidismo en España? Una primera lectura — superficial — de los resultados parece darnos una respuesta indiscutiblemente afirmativa. Desde luego, el sistema de partidos surgido de los dos últimos procesos electorales ya no puede calificarse de bipartidista.

Sin embargo, la cuestión es mucho más compleja. En un plano técnico-electoral, nos podemos plantear si la fragmentación de la representación que se ha producido - y que entra en contradicción con un sistema electoral pensado precisamente para favorecer el efecto contrario- se puede mantener en el tiempo, lo que nos llevaría a plantearnos también el problema de la gobernabilidad a medio plazo y a intentar aventurar posibles soluciones legislativas para este desde una óptica de ingeniería electoral ${ }^{1}$.

No es la intención de esta breve nota dar una respuesta inequívoca a este interrogante. Probablemente, en estos momentos nadie pueda hacerlo. Por ello, me planteo un objetivo mucho más modesto, que consiste sencillamente realizar un breve análisis del funcionamiento del sistema electoral observando su impacto real sobre los resultados de 2015 y 2016, y aventurar alguna reflexión sobre su posible influencia en la evolución futura del sistema de partidos.

\section{LOS EFECTOS DEL SISTEMA ELECTORAL Y SU IMPACTO EN LOS RESULTADOS DE 2015 Y 2016}

\section{UN SISTEMA ELECTORAL PENSADO PARA FABRICAR MAYORÍAS Y, ESPECIALMENTE, MAYORÍAS CONSERVADORAS}

Partiendo de la base de que «la falta de inocencia de los sistemas electorales es un hecho más que comprobado y asumido» (Soriano y Alarcón, 2001:

\footnotetext{
La ingeniería electoral, como parte de la ingeniería constitucional, parte de la idea de que los sistemas electorales configuran los sistemas de partidos y pueden cambiarlos, de manera que a través del diseño de un sistema electoral u otro se puede contribuir a la consolidación de un sistema de partidos óptimo. Véase Sartori (1999: 84); y, para una visión más amplia, véase Sartori (1994).
} 
118), no se puede abordar el análisis de los efectos de nuestro sistema electoral sin tomar plena conciencia de la intencionalidad con la que se diseñó. El sistema electoral del Congreso de los Diputados, que se remonta a la Ley 1/1977, de 4 de enero, para la Reforma Política, y al Real Decreto-Ley 20/1977, de 18 de marzo, que reguló las primeras elecciones democráticas, se diseñó en la Transición con la intención inequívoca de favorecer la concentración de la representación, para evitar la excesiva fragmentación del parlamento y propiciar, de este modo, la estabilidad gubernamental y la consolidación de un sistema de partidos fuerte integrado por un número no excesivamente alto de formaciones bien cohesionadas, objetivos de vital importancia en el período fundacional de la democracia (Rubio Llorente, 2014: 143). También se diseñó con un fuerte sesgo conservador, claramente orientado a «ayudar» a la opción política pilotada desde el Gobierno de Suárez (Urdánoz, 2008: 58-60). Los principales instrumentos de que se dispusieron para el logro de tales objetivos fueron la opción por un sistema de representación proporcional, pero con circunscripciones provinciales de pequeña magnitud de escaños y la atribución de un mínimo de dos diputados a cada circunscripción ${ }^{2}$. Además, también se añadió la barrera electoral del $3 \%$ en cada circunscripción, aunque de escasa o casi nula operatividad, salvo eventualmente en las circunscripciones más grandes. Este diseño fue fruto de una transacción en la que el Gobierno de Suárez trató de contentar por una parte a los partidos de la oposición democrática, que defendían la representación proporcional, y por otra parte a un cierto sector del régimen franquista, presente en las Cortes y que, más tarde, se agruparía en AP, que defendía un sistema mayoritario como medio para favorecer un ejecutivo fuerte (Montero y Riera, 2009: 381).

El resultado de ese diseño es un sistema electoral que Óscar Alzaga, uno de los expertos que participó en la redacción de la normativa electoral de 1977, llegó a calificar de "maquiavélico»" en el que el que las provincias menos

2 Téngase en cuenta que el mínimo de dos escaños por provincia no estaba previsto ni en el anteproyecto de la Ley para la Reforma Política, del presidente de Las Cortes, ni en el proyecto del Gobierno. Fue fruto de una enmienda in voce, presentada por un procurador en el debate plenario, basada sobre todo en argumentos de equilibrio de la representación territorial. Aceptada esta enmienda, la Ley para la Reforma Política prevé que exista un mínimo provincial, pero no lo concreta. La concreción llegará con el Decreto-ley de 1977 (Fernández-Miranda Campoamor, 2009: 27-28).

3 Alzaga ha admitido que el objetivo buscado era que UCD alcanzase la mayoría absoluta de los escaños solo con un 36-37 \% de los votos. El resultado final no estuvo muy lejos: UCD obtuvo el 34,44\% de los votos y con 165 diputados se quedó a once escaños de la mayoría absoluta (cit. por Torres del Moral, 2009: 66 y ss.). 
pobladas tienen un peso desproporcionado y en el que en dos tercios de las circunscripciones no existe realmente la proporcionalidad, lo que castiga — casi se podría decir que con ensañamiento- a las fuerzas minoritarias de implantación nacional. La consecuencia es, pues, que «la competición electoral en España no tiene lugar en condiciones iguales en todas las circunscripciones, y los votos no cuentan todos lo mismo» (Penadés y Santiuste, 2013: 90).

En España, hay en la actualidad 33 provincias en las que se reparten seis escaños o menos, en las que, por consiguiente, la barrera natural —que está en función del tamaño de la circunscripción (Lijphart, 1995: 25 y ss.) es superior en la mayor parte de las circunstancias al $15 \%$ de los votos. No es de extrañar, por tanto, que Nohlen lo considere como un ejemplo atípico de «sistema de mayoría» en el que la regla decisoria de la proporcionalidad se inserta en un sistema electoral cuya orientación y efecto es la representación por mayoría (Nohlen, 2013: 22). Aun así, también se ha destacado que, aunque la proporcionalidad en los distritos de pequeña magnitud sea limitada, ello no ha impedido que haya competencia de varias candidaturas y que se haya permitido la representación de varios partidos (López Nieto, 2016: 75).

En realidad, puede decirse que en España no hay un solo sistema electoral, sino dos o tres (Penadés y Santiuste, 2013), en función de la magnitud de las circunscripciones, lo cual plantea un problema jurídico-constitucional que no es baladí, pues, aunque la coexistencia de sistemas electorales diferentes no es per se inconstitucional cuando está justificada, por ejemplo, por la necesidad de dotar de una representación diferenciada a unidades territoriales específicas, en el caso del sistema electoral del Congreso de los Diputados, no hay ninguna finalidad constitucionalmente legítima que justifique la posición desigual de los ciudadanos ante efectos tan dispares del sistema en las distintas provincias (Sánchez Muñoz, 1999: 524 y ss.; Mora Molina, 2013: 95). Lo que sucede, por decirlo de manera sencilla, es que unos españoles votan con un sistema proporcional y otros con un sistema cuasimayoritario. Esto supone, en términos más técnicos, que se produce una desigualdad en cuanto a la vulnerabilidad del voto (Urdánoz, 2008: 60). Cuando un elector, como consecuencia del sistema, se ve impelido a votar por un partido que no es su primera opción, entonces su voto es más vulnerable.

Ciertamente, es preciso tener en cuenta que la opción por la provincia como circunscripción electoral es una alternativa consciente del constituyente y que, evidentemente, dicha opción tiene una ratio: una ratio que no es otra que plasmar el compromiso entre las posiciones de la derecha salida del régimen franquista y la oposición democrática, al que he hecho referencia anteriormente. Otra cosa es que esa ratio siga teniendo sentido en la España actual 
— que a mi juicio no lo tiene-y que las distorsiones que el sistema provoca no sean cada vez más una fuente de cuestionamiento de su legitimidad.

A la hora de analizar los efectos que ha tenido el sistema electoral en España, aun admitiendo que, como recalca Rae, "la influencia del sistema electoral sobre sistema de partidos a nivel parlamentario es generalmente menos importante que muchas otras fuerzas que se expresan en los resultados electorales» (1977: 100), lo cierto es que en nuestro país el sistema sí ha tenido una influencia muy decisiva, hasta el punto de que estamos, como señala Presno Linera, ante un "caso paradigmático en el Derecho comparado» de influencia del sistema electoral sobre el sistema de partidos (2015: 14).

Los efectos sumados de concentración de la representación y de desproporcionalidad a favor del partido ganador $y$, en menor medida, del segundo partido han funcionado de manera muy efectiva hasta 2015, lo que ha propiciado la creación de mayorías parlamentarias sólidas que encajan perfectamente en la definición de «mayorías fabricadas» $\mathrm{o}$ «manufacturadas» (manufactured majorities), que en su día hizo el propio Rae para referirse a las mayorías construidas por el sistema electoral, por contraposición a las «mayorías ganadas» (earned majorities), que se deben al voto de los electores (Rae, 1977: 76). No cabe duda de que estos «efectos mecánicos» del sistema se han visto reforzados también por sus «efectos psicológicos»" ${ }^{4}$, es decir, en este caso concreto por la tendencia de los electores a votar de forma estratégica, haciéndose eco de las interesadas llamadas al voto útil por parte de los grandes partidos (Montero y Riera, 2009: 397)5.

Así, si comparamos el porcentaje de votos obtenidos por los dos primeros partidos en cada una de las convocatorias electorales con el porcentaje de escaños atribuidos por el sistema en el Congreso de los Diputados, podemos observar el efecto de desproporcionalidad que se produce (TABLA 1).

El efecto de desproporcionalidad derivado de la existencia de un número elevado de circunscripciones de pequeña magnitud se amplifica por la desigualdad de representación entre las provincias más y menos pobladas, que, como se ha avanzado antes, es consecuencia de la atribución de un mínimo de dos escaños a cada provincia. Como explican con claridad Montero y Riera (2009: 403), como resultado de la combinación de ese mínimo provincial con un Congreso de tamaño no demasiado grande, solo se reparten 248 escaños (entre 50 circunscripciones) atendiendo a criterios de población, lo que otorga una notable sobrerrepresentación a las circunscripciones menos pobladas.

4 Sobre la clásica distinción entre los efectos mecánicos y psicológicos de los sistemas electorales, véase Duverger (2001: 252).

5 Como estudios específicos sobre el voto estratégico, véase Moreno y Oñate (2004); García Viñuela y Artés (2009). 
EL FIN (MOMENTÁNEO) DEL BIPARTIDISMO EN ESPAÑA: ANÁLISIS DE LOS RESULTADOS... 245

TABLA 1. Diferencias entre el porcentaje de votos y escaños del partido ganador en las elecciones entre 1977 y 2011

\begin{tabular}{ccccc}
\hline Convocatoria & 1. $^{\text {er }}$ partido & Diferencia & 2. $^{\circ}$ partido & Diferencia \\
\hline 1977 & UCD & $+12,9$ & PSOE & $+4,4$ \\
1979 & UCD & $+12,9$ & PSOE & $+4,1$ \\
1982 & PSOE & $+10,4$ & AP-PDP & $+4,7$ \\
1986 & PSOE & $+8,5$ & AP-PDP-PL & $+3,9$ \\
1989 & PSOE & $+10,4$ & PP & $+4,8$ \\
1993 & PSOE & $+6,0$ & PP & $+5,5$ \\
1996 & PP & $+5,7$ & PSOE & $+2,8$ \\
2000 & PP & $+7,8$ & PSOE & $+1,6$ \\
2004 & PSOE & $+4,2$ & PP & $+4,6$ \\
2008 & PSOE & $+4,6$ & PP & $+3,6$ \\
2011 & PP & $+8,5$ & PSOE & $+2,7$ \\
\hline
\end{tabular}

Fuente: Montero y Riera (2009: 400) —hasta 2008—, y elaboración propia.

En mi opinión, estas diferencias de representación entre provincias afectan al principio de igualdad del voto, pues se rompe la regla del equilibrio demográfico entre circunscripciones que exige que cada representante sea elegido por un número igual de electores (Sánchez Muñoz, 1999: 503-515) . Nuestro sistema entra así en contradicción con los estándares internacionales establecidos en el Documento de Copenhague de la Organización para la Seguridad y la Cooperación en Europa (OSCE) (párrafo 7.3) ${ }^{7}$ y en el Código de Buenas Prácticas en Materia Electoral ${ }^{8}$ de la Comisión de Venecia, que recomienda expresamente que "los escaños deberán repartirse por igual entre las circunscripciones» (Sección I.2). Esta contradicción ha sido recordada repetidamente por los informes de evaluación elaborados por las misiones de observación de la OSCE sobre diversos procesos electorales en España?a. De

6 Una recopilación de opiniones doctrinales en relación con la desigualdad de nuestro sistema electoral, incluyendo citas de Martínez Cuadrado, Nohlen y Schultze, Álvarez Conde y González Hernández, Fernández Segado, Santolaya Machetti, Garrorena Morales y García Morillo, se encuentra en Urdánoz (2009: 272-273).

Aprobado el 29 de junio de 1990.

8 Aprobado por la Comisión de Venecia en su 52.a Sesión (18-19 de octubre de 2002).

9 Véase, por ejemplo, los Informes Finales sobre las Elecciones Generales de 20 de noviembre de 2011 y de 20 de diciembre de 2015 en los que se dice textualmente que «El reparto de escaños por circunscripciones no asegura completamente la igualdad de voto, con arreglo a lo dispuesto en el art. 7.3 del Documento de Copenhague» (p. 1). 
hecho, el sistema electoral español ocupa la primera posición en Europa y una de las primeras del mundo en cuanto a malapportionment; esto es, la medida que cuantifica la distorsión existente entre el porcentaje de escaños que se otorgan a las circunscripciones respecto a la población que reside en ellas (Fernández Esquer, 2015; Urdánoz, 2009: 280), aunque, por otra parte, es preciso señalar que también se han alzado autorizadas voces en la doctrina en defensa de la opción del constituyente, reforzada por el legislador, para favorecer la representación diferenciada de todas las provincias (Fernández-Miranda, 2009: 28 y ss.; Delgado-Iribarren, 2012: 16-17).

Pero lo realmente peculiar del sistema electoral español, como han apuntado con acierto Penadés y Santiuste, no es solo que haya circunscripciones que aplican un sistema cuasimayoritario y otras que aplican un sistema proporcional, sino que las zonas de sistema mayoritario, además, coinciden en ser también zonas sobrerrepresentadas. De este modo, los dos mecanismos de ventaja, el reparto mayoritario y la sobrerrepresentación, se acumulan sobre los ganadores en ciertos distritos (Penadés y Santiuste, 2013: 106). Dicho más claramente, en las circunscripciones pequeñas los partidos ganadores resultan doblemente aventajados. En primer lugar, reciben una importante ventaja sobre sus competidores locales debido al carácter mayoritario del reparto de escaños. En segundo lugar, al tratarse de circunscripciones sobrerrepresentadas, reciben una nueva ventaja con respecto a los que los obtienen en otras zonas (Penadés y Santiuste, 2013: 110).

A todo lo dicho hasta ahora hay que sumarle que el fenómeno de la desigualdad de la representación en España tiene un elemento añadido que lo convierte en un factor de distorsión del resultado electoral especialmente censurable. El problema no es solo que exista desigualdad, sino que dicha desigualdad no es neutral desde un punto de vista de la competición entre los partidos, lo que justifica que se hablemos de un sesgo o incluso, por qué no llamar a las cosas por su nombre, de una especie de gerrymandering.

Observando de nuevo la TABLA 1 puede comprobarse también que, en términos generales, el efecto de sobrerrepresentación que favorece a los dos primeros partidos es relativamente mayor a favor de los partidos de centro-derecha, y esto es debido precisamente a lo que hemos denominado antes el sesgo conservador del sistema (Montero y Riera, 2009: 404).

Penadés y Santiuste han comparado en detalle cómo han afectado los efectos de desproporcionalidad y de desigualdad de la representación a los dos primeros partidos entre 1977 y 2011 y han llegado a la conclusión inequívoca de que los partidos de centro-derecha (UCD en su momento y posteriormente $\mathrm{PP})$ siempre se han beneficiado de estos efectos en mayor medida que el PSOE, tanto cuando han sido los partidos más votados, como cuando han sido la 
segunda fuerza política (Penadés y Santiuste, 2013: 110 y ss.). Por decirlo de forma sencilla, las fuerzas de centro-derecha suelen ganar más en las circunscripciones cuasimayoritarias que además están sobrerrepresentadas, obteniendo así un doble premio en términos de representación parlamentaria.

Esto es así porque los habitantes de las provincias menos pobladas tienen unas características sociológicas particulares que los predisponen a apoyar a fuerzas políticas más conservadoras, básicamente porque se trata de una población rural en mayor medida y de población más envejecida. El legislador de 1977 lo vio con suma claridad: cuando se introdujo el mínimo provincial de dos escaños, la oposición democrática de izquierdas tenía una implantación sobre todo entre la población joven y urbana. Por consiguiente, la desigualdad del voto beneficiaba claramente a las fuerzas de centro-derecha.

Tras la victoria electoral del PSOE en 1982, este partido se dio cuenta de que, debido a su fuerte implantación en las provincias rurales del sur de España, la desigualdad del sistema también le beneficiaba. Por ello, la Ley Orgánica del Régimen Electoral General (LOREG) de 1985 mantuvo el mínimo de dos diputados por provincia.

En tiempos más recientes, el sesgo conservador ha seguido funcionando en el mismo sentido que en 1977 e, incluso, podríamos decir que se han reforzado sus efectos, pues muchas de las provincias cuya población era baja en 1977 han perdido todavía más población y ahora está relativamente más envejecida. El partido que sale más beneficiado es indudablemente el PP. Solo hay que echar un vistazo a los resultados de las nueve provincias de Castilla y León — sin duda, el ejemplo paradigmático — para darse cuenta de ello. En las elecciones de 2016, el PP, con 643093 votos en las provincias de Castilla y León, obtuvo dieciocho diputados. En Madrid, con 1325665 votos, solo obtuvo quince. Pero el PSOE también sale relativamente beneficiado, aunque mucho menos, como veremos a continuación cuando analicemos los datos de su pugna particular con Podemos en las dos últimas convocatorias electorales.

\section{2. ¿QUÉ HA PASADO EN 2015 Y 2016?}

$\mathrm{Si}$, como acabamos de ver, el sistema electoral ha fabricado mayorías eficazmente hasta 2011, nos podemos plantear ahora cómo han funcionado los efectos del sistema electoral en 2015 y 2016.

A primera vista, podría parecer que el sistema electoral ha fracasado o no ha cumplido con lo que se esperaba de él, pues un sistema electoral pensado para producir un efecto — reducción de la fragmentación de la representación y premio a los partidos mayoritarios para favorecer la gobernabilidad- ha 
producido justamente el efecto contrario, pues lo que tenemos es un parlamento fragmentado y una muy difícil gobernabilidad. Sin embargo, los efectos del sistema han sido exactamente los mismos de siempre, como veremos a continuación.

Si observamos la TABLA 2, veremos que el efecto de desproporcionalidad ha seguido funcionando a favor de los dos primeros partidos en términos muy similares a los de convocatorias anteriores, sin embargo, el efecto no es tan fuerte como para poder fabricar una mayoría parlamentaria sólida cuando el apoyo electoral al partido mayoritario desciende por debajo de ciertas cotas.

Tabla 2. Diferencias entre el porcentaje de votos y escaños de los principales partidos politicos de ámbito nacional en las elecciones de 2015 y 2016

\begin{tabular}{lcc}
\hline & 2015 & 2016 \\
\hline PP & $+6,4$ & $+6,1$ \\
PSOE & $+3,7$ & $+1,7$ \\
Podemos* & $-0,97$ & \\
Ciudadanos & $-2,5$ & $-3,92$ \\
UP (IU) & $-3,11$ & \\
Unidos Podemos** & & $-0,86$ \\
\hline
\end{tabular}

Fuente: elaboración propia.

* Incluye: Podemos, En Comú-Podem, Podemos-Compromis y Podemos-En MareaAnova-EU.

** Incluye: Unidos Podemos (Podemos-IU-Equo), En Comú-Podem, Podemos-CompromisEUPV y Podemos-En Marea-Anova-EU.

Hay varios datos que llaman la atención y que requieren una explicación más detallada. El primero es el hecho de que el PP, a pesar de haber obtenido un $33,01 \%$ de los votos en las elecciones de 2016 - por tanto, un resultado próximo al 34,44\% que obtuvo la UCD en 1977-no se ha beneficiado de un efecto de desproporcionalidad tan acusado como en aquella ocasión, que le permitió a la UCD obtener 165 escaños y formar Gobierno sin dificultad. Hay dos factores que, combinados, explican esta diferencia entre ambas situaciones.

El primero es la distribución del voto entre los demás partidos. En 1977, la tercera y cuarta fuerzas políticas (PCE y AP) obtuvieron solo 1,7 y 1,5 millones de votos, respectivamente, lo que dificultó su acceso a escaños en gran parte de las circunscripciones. En 2016, en cambio, la tercera fuerza 
(Unidos Podemos y sus confluencias) ha obtenido 5,1 millones de votos y la cuarta fuerza (Ciudadanos) ha logrado 3,1 millones, lo que ha permitido que estos partidos hayan podido acceder a la representación en circunscripciones de menor magnitud.

El segundo factor es la distribución geográfica del voto. En 1977, la UCD tenía un apoyo electoral mucho más localizado en los distritos pequeños en comparación con los grandes (Penadés y Santiuste, 2013: 112) y, por ello, fue capaz de maximizar el sesgo conservador derivado de la desigualdad de la representación a favor de las provincias menos pobladas obteniendo una cosecha de escaños espectacular en la España rural. Esta pauta de localización del voto de centro-derecha se ha ido suavizando con el tiempo y hoy en día ya no es tan marcada. Por citar los ejemplos más significativos, en 1977 solo en las provincias que hoy forman las comunidades de Galicia, Castilla y León y Extremadura, la UCD obtuvo 53 escaños. En 2016, el PP, a pesar de haber ganado en dichas comunidades, solo ha podido obtener 35 escaños en ellas.

El segundo hecho significativo que también requiere una explicación detallada es la influencia del efecto de desproporcionalidad del sistema electoral en las pugnas particulares que han protagonizado, en el espacio del centro-derecha, PP y Ciudadanos; y en el espacio de centro-izquierda, PSOE y Podemos.

En el primero de los dos enfrentamientos, como acabamos de ver en la TABLA 2, el efecto de desproporcionalidad del sistema ha premiado significativamente al PP y ha castigado, también significativamente, a Ciudadanos, de tal forma que la representación parlamentaria finalmente obtenida magnifica la distancia real existente entre ambas fuerzas. En 2015, con un 28,71 \%, el PP obtiene 123 diputados; y Ciudadanos, con un 13,94\%, que es casi la mitad, se queda solo con 40 . La desproporción se aprecia con claridad cuando se observa que cada diputado le cuesta al PP 58837 votos, mientras que a Ciudadanos le supone 87863 votos. Un reparto estrictamente proporcional entre ambas fuerzas habría dado como resultado 100-101 escaños para el PP y 49 para Ciudadanos. En 2016, el efecto de desproporcionalidad se acentúa aún más. El PP, con un 33,01 \% de los votos, obtiene 137 diputados; y Ciudadanos, con un 13,06 \% de los votos, obtiene tan solo 32. Esto significa que cada diputado le cuesta al primero 57965 votos; y al segundo, 98174 y que, con un hipotético reparto estrictamente proporcional, el primero se habría adjudicado 115-116 escaños, mientras que el segundo habría obtenido 46.

En el segundo de los enfrentamientos, el que opone a PSOE y Podemos, resulta especialmente reveladora la diferente manera en que ambas fuerzas políticas se ven afectadas por el efecto de desproporcionalidad del sistema, a pesar de haber obtenido porcentajes de voto muy similares. Como puede 
comprobarse observando de nuevo la TABLA 2, el PSOE se ve beneficiado por el efecto de desproporcionalidad del sistema, más intensamente en 2015, menos en 2016, mientras que Podemos (y sus confluencias) se ve ligeramente perjudicado en ambas convocatorias. Ciertamente, consigue corregir algo ese efecto negativo mediante la coalición con Izquierda Unida en 2016, pero de manera prácticamente imperceptible.

La explicación de este hecho es sencilla y tiene que ver con la manera en que ambas fuerzas políticas se ven afectadas por lo que hemos venido llamando el sesgo conservador derivado de la sobrerrepresentación de las provincias menos pobladas. Lo que ha pasado es, simplemente, que el PSOE se ha comportado mejor electoralmente en estas provincias, algo que se explica por la diferente estructura del voto que se ha estudiado entre PSOE y Podemos en términos de edad, nivel de educación, uso de nuevas tecnologías y residencia en zonas urbanas o rurales ${ }^{10}$.

Si tomamos los resultados electorales comparados de PSOE y Podemos en las elecciones de 2015 y 2016 en las circunscripciones de magnitud igual o inferior a seis escaños ${ }^{11}$, obtenemos resultados muy significativos y bastante similares entre las dos convocatorias.

Efectivamente, en este conjunto de provincias, el comportamiento electoral del PSOE fue mejor que el de Podemos, que obtuvo en el conjunto de todas ellas 1976096 votos en 2015 y 1880616 votos en 2016, frente a 1328494 y 1357643 votos, respectivamente, de la formación morada. Estos resultados le permitieron al PSOE obtener 42 escaños en estas provincias en 2015 y 40 en 2016, mientras que Podemos solo obtuvo veintidós escaños en ambas convocatorias. Esto coincide con las variables sociológicas que hemos citado anteriormente en cuanto a la estructura del voto de ambos partidos.

Así pues, debido a estos mejores resultados en las provincias menos pobladas, el PSOE ha podido beneficiarse del sesgo conservador del sistema electoral en términos relativos frente a Podemos. El resultado de ello es que, volviendo a los datos globales del conjunto de España, el PSOE en 2015 tuvo

10 Como ejemplo se puede citar el Barómetro del CIS de octubre de 2016 (estudio 3156) que muestra que el apoyo electoral al PSOE es significativamente menor en ciudades de más de 400000 habitantes, en menores de cuarenta y cuatro años, y entre estudiantes, titulares superiores, profesionales y cuadros medios, categorías todas que, a su vez, tienden a residir en provincias con más población.

11 Álava, Albacete, Almería, Ávila, Badajoz, Burgos, Ciudad Real, Cáceres, Cantabria, Castellón, Córdoba, Cuenca, Girona, Guadalajara, Guipúzcoa, Huelva, Huesca, Jaén, La Rioja, León, Lleida, Lugo, Navarra, Orense, Palencia, Salamanca, Segovia, Soria, Tarragona, Teruel, Toledo, Valladolid y Zamora. 
una ratio de votos por diputado de 61614 , mientras que en el caso de Podemos esta ratio fue de 75 546. En 2016, los datos fueron similares: una ratio de 64045 votos por diputado para el PSOE y de 71655 votos por diputado para Podemos. En definitiva, el PSOE, merced a su mejor posicionamiento en las provincias menos pobladas, derivado de su mayor apoyo entre la población de más edad y entre la población rural, puede obtener más diputados con menos votos. Nótese, no obstante, que el efecto beneficioso del sesgo conservador para el PSOE ha sido menor en 2016 que en 2015, debido a que en el grupo de provincias con seis escaños o menos los socialistas han perdido 95480 votos, lo que le ha hecho perder dos escaños, mientras que Podemos, probablemente como consecuencia del pacto con Izquierda Unida, ha crecido en ese mismo grupo de provincias en 29149 votos, manteniendo su representación en escaños.

Como recapitulación de este apartado se pueden establecer una serie de conclusiones provisionales sobre los efectos del sistema electoral en las elecciones de 2015 y 2016:

1. Pese a su descenso en el número de votos, los dos primeros partidos (PP y PSOE) se siguen beneficiando del efecto de desproporcionalidad del sistema, que sigue perjudicando a las demás fuerzas políticas de ámbito nacional.

2. El sistema no ha podido fabricar una mayoría parlamentaria más sólida debido a que el PP, aun beneficiándose del efecto de desproporcionalidad y del sesgo conservador del sistema, no llega a maximizar ese beneficio en los niveles que consiguió la UCD en los años 1977 y 1979.

3. En el espacio del centro-derecha, el efecto de desproporcionalidad del sistema ha premiado muy intensamente al PP y ha castigado, también muy intensamente, a Ciudadanos, que es la fuerza política más perjudicada por el sistema en términos generales.

4. En la particular pugna por ocupar el papel central en el polo de la izquierda, el PSOE se ha beneficiado, frente a Podemos, del sesgo conservador del sistema, pero de forma muy exigua, si lo comparamos con el beneficio que obtiene el PP.

\section{LA POSIBLE INFLUENCIA DEL SISTEMA ELECTORAL EN LA EVOLUCIÓN FUTURA DEL SISTEMA DE PARTIDOS... Y VICEVERSA}

Partiendo de los datos analizados en el apartado anterior, constatamos que nuestro sistema electoral, que había estado favoreciendo el bipartidismo 
durante casi cuatro décadas y fabricando mayorías parlamentarias estables, como consecuencia del terremoto electoral de 2015 y de su réplica de 2016, ha dado como resultado una situación de «multipartidismo fragmentado» (Fernández Esquer, 2016).

Como señalan Penadés y Pavía, las elecciones de 2015 - y podríamos añadir que también las de 2016 - han demostrado que con el mismo sistema electoral puede haber cuatro partidos que superen el 18-20\% de los votos y que, más o menos (dejando de lado los premios y castigos del sistema) pueden obtener una representación acorde con esa fuerza electoral (2016: 26).

Sin embargo, cabe plantearse si es posible que este nuevo sistema de partidos, surgido coyunturalmente de una situación de confluencia entre una crisis económica profunda y una creciente desafección ciudadana hacia la política, sea sostenible en el tiempo, y qué influencia podrá tener el propio sistema electoral en dicha sostenibilidad. Por otro lado, en sentido inverso, cabe también cuestionarse si la aparición de este nuevo sistema de partidos puede tener una influencia en la transformación del propio sistema electoral y, si es así, en qué sentido.

La ciencia política ha relativizado enormemente la idea de que el sistema de partidos es un producto del sistema electoral, que parecía estar en la base de las célebres Leyes de Duverger (Duverger, 1950: 13), aunque ciertamente ya el propio autor había matizado mucho este determinismo en diferentes versiones de sus trabajos (Nohlen, 2013: 11 y ss.). La experiencia de las elecciones de 2015 y 2016 demuestra precisamente que la influencia de los sistemas electorales es relativa y que los factores puramente políticos tienen un peso fundamental en las transformaciones de los sistemas de partidos. Ahora bien, aunque relativa, la influencia del sistema electoral existe y la experiencia electoral de 2015 y 2016, lejos de desmentir dicha influencia, la confirma, pues el sistema ha contribuido significativamente a amortiguar la caída de los dos principales partidos y, muy en especial, del PP.

Evidentemente, el futuro del sistema de partidos en España no va a estar exclusivamente condicionado por el sistema electoral, pero eso no quiere decir que el sistema electoral no vaya a tener cierta influencia o que no vaya a influir en las decisiones que tendrán que adoptar los actores políticos.

Desde este punto de vista, según ciertas opiniones, estamos ante un «nuevo equilibrio» (Penadés y Pavía, 2016: 26) y el sistema electoral, que ha permitido llegar a este nuevo equilibrio, no va a forzar un cambio en el sistema de partidos, lo que no quiere decir que este no cambie por otros motivos. En otras palabras, según ese punto de vista, el funcionamiento de las reglas del sistema no va a suponer un incentivo para que los líderes políticos decidan reorganizar la oferta de partidos (más allá de lo que supone la alianza Podemos-IU) 
o para que los votantes decidan votar estratégicamente y concentrar su apoyo en ciertas fuerzas políticas.

Aunque es extraordinariamente arriesgado hacer previsiones en un contexto político tan cambiante y es sumamente fácil deslizarse por la pendiente del wishful thinking, a mi juicio, no está tan claro que ese pretendido nuevo equilibrio haya llegado; de hecho, por el contrario, el mantenimiento a largo plazo del multipartidismo surgido de las urnas en 2015 y 2016 no parece sostenible. Desde luego, si el sistema electoral va a influir en algo en la evolución futura, será en el sentido de que las aguas vuelvan a un cierto cauce bipartidista, aunque conviniendo en que su influencia no será absolutamente determinante. De hecho, los resultados de 2016, en comparación con los de 2015, parecen ir en esta dirección.

De una parte, ante una previsible recuperación del PP, de mantenerse la tendencia apuntada con las elecciones de 2016, no parece posible que se mantenga una división en el espacio de centro-derecha con dos partidos fuertes. En las dos últimas convocatorias, el sistema electoral ha castigado a Ciudadanos más que a ningún otro partido y ha premiado al PP más que a ningún otro partido. Esta ventaja relativa del PP se reforzará aún más si Ciudadanos sigue perdiendo apoyos y a los efectos mecánicos del sistema se pueden sumar pronto los efectos psicológicos (voto estratégico) si se mantiene esa tendencia.

De otra parte, aunque es más imprevisible lo que pueda suceder en el espacio del centro-izquierda, no parece tampoco sostenible que se mantenga una división entre dos partidos (PSOE y Podemos) con un apoyo electoral similar (en torno al $20 \%$ ). Lo que parece probable, en cambio, es que, en esta pugna particular, uno de los dos partidos acabe imponiéndose al otro como el principal referente en el espacio de la izquierda y, en ese contexto, el sistema electoral con su efecto de desproporcionalidad acabará reforzando esa tendencia. Como acabamos de ver, de momento el PSOE es el partido que sale más beneficiado por el sistema electoral, pero la ventaja relativa que obtiene como consecuencia del sesgo conservador es muy exigua, aunque le haya servido en las últimas convocatorias para mantener el tipo ante el reto que supone el avance electoral de Podemos.

No hay que olvidar tampoco que, además del sistema electoral, existen otros elementos que forman parte de la legislación electoral de contorno y que también pueden causar un efecto significativo sobre la evolución del sistema de partidos. El principal de ellos es la normativa sobre financiación de los partidos. A este respecto, es preciso tener en cuenta que la financiación de los partidos políticos en España es fundamentalmente pública (en más de un 80 $\%$ en algunos casos) y que la representación parlamentaria es uno de los factores fundamentales en el reparto de las subvenciones públicas (exclusivo en 
el caso de las subvenciones electorales). Estos criterios de reparto suponen una amenaza para la igualdad de oportunidades (Sánchez Muñoz, 2007: 231), pues dificultan enormemente la competitividad de los partidos pequeños de implantación nacional. Por consiguiente, la sostenibilidad del sistema de partidos surgido de las elecciones de 2015 y 2016 también se va a ver condicionada por la capacidad de los nuevos actores, Podemos y Ciudadanos, de asegurarse unas fuentes de financiación estables. En el caso específico de Ciudadanos, debido a su menor representación parlamentaria, las normas de reparto de la financiación pública no lo van a ayudar a conseguir ese objetivo, sino todo lo contrario.

Si es importante plantearse cómo va a afectar el sistema electoral a la evolución del sistema de partidos, no lo es menos plantearse la cuestión inversa, es decir, plantearse hasta qué punto la nueva configuración parlamentaria puede propiciar un cambio de las reglas del juego electoral, teniendo en cuenta que esas reglas del juego son definidas precisamente por los propios jugadores.

En este orden de ideas, es necesario analizar en primer lugar cuáles son las propuestas de reforma que han puesto sobre la mesa los distintos actores y las posibilidades de que dichas propuestas se puedan realizar en la práctica.

Tanto Podemos como Ciudadanos han llevado en sus programas electorales propuestas de reforma del sistema electoral. Podemos básicamente ha planteado la sustitución de las circunscripciones provinciales por autonómicas y la utilización de la fórmula de la media mayor para mejorar la proporcionalidad ${ }^{12}$ y Ciudadanos ha propuesto «un sistema electoral de doble lista desbloqueada para la elección uninominal y proporcional de ámbito territorial y nacional, respectivamente» $(\text { sic. })^{13}$, lo cual parece inspirarse en el modelo alemán, con un reparto proporcional a nivel nacional, pero con una parte de los diputados elegidos en circunscripciones uninominales ${ }^{14}$. No se especifica si se propone el establecimiento de una cláusula de barrera nacional, pero se puede intuir que sí. Por su parte, el programa electoral del PSOE habla de listas desbloqueadas y de «mejorar la proporcionalidad $»^{15}$, pero no precisa si ello implica

12 Programa electoral de Podemos para las elecciones de 2016, medida 228. Disponible en: https://lasonrisadeunpais.es/programa/

13 Programa electoral de Ciudadanos para las elecciones de 2016, medida 277. Disponible en: https://www.ciudadanos-cs.org/programa-electoral

14 Sobre el sistema alemán y su posible implantación en España: Vidal Prado (2012). Sobre la reforma reciente de este sistema, véase Vázquez Lapuente y Jiménez Seral (2014).

15 Programa electoral del PSOE para las elecciones de 2016, p. 76. Disponible en: http://www.psoe.es/media-content/2016/05/PSOE-Programa-Electoral-2016.pdf 
el cambio de la circunscripción o no. En momentos anteriores de mayor precisión programática, este partido flirteó con el modelo alemán, pero no hay una postura clara al respecto. Por último, el programa del PP sencillamente no contiene ninguna referencia a la reforma del sistema electoral de las elecciones legislativas.

Con este grado de disparidad entre las propuestas no parece una tarea fácil lograr el consenso necesario para emprender una reforma del sistema electoral, y mucho menos si esa reforma implica la reforma del art. 68 de la Constitución española para modificar la circunscripción electoral. Una hipotética suma de diputados de PSOE, Podemos y Ciudadanos podría reformar la LOREG, pero no es fácil imaginar cuál podría ser el común denominador entre estas tres fuerzas, más allá de mitigar los efectos del sesgo conservador mediante una reducción del mínimo provincial de dos a uno. Sin embargo, esta reforma, que podría ser interesante para Podemos y Ciudadanos, no lo sería tanto para el PSOE que, como hemos visto, se ha beneficiado de este sesgo conservador en su competición particular con Podemos.

Además, habría que valorar que esta medida, incluso si va acompañada de un aumento del número de diputados a cuatrocientos, tiene implicaciones negativas en términos de proporcionalidad en las circunscripciones de menor población - a pesar de la mejora relativa que se apreciaría en los índices de proporcionalidad global del sistema- ${ }^{16}$, lo que nos conduciría a lo que Urdánoz ha calificado como «la paradoja de las desigualdades» (2009: 286): reducir la desigualdad del valor del voto (reduciendo de dos a uno el mínimo provincial) supondría incrementar la desigualdad en cuanto a los efectos del sistema en las diferentes circunscripciones, pues algunas de ellas serían más proporcionales, pero otras, en cambio, seguirían siendo mayoritarias e, incluso, lo serían mucho más (piénsese, por ejemplo, en las circunscripciones que verían reducida su magnitud a uno o dos escaños). Esto último, además, seguiría introduciendo un cierto sesgo conservador en el sistema (Montero y Riera, 2009: 410 y ss.).

El partido más interesado en la reforma es, desde luego, Ciudadanos, puesto que es el partido más perjudicado por el sistema actual. Sin embargo, no parece que, con la configuración actual del parlamento, tenga fuerza suficiente para convencer a los demás partidos de la necesidad de esta reforma. En el acuerdo «150 compromisos para mejorar España», que es la base del apoyo de Ciudadanos a la investidura de Mariano Rajoy, lo máximo que ha podido

16 Solo si se incluyese también en la reforma la adopción de la formula Hare se produciría una reducción significativa de la desproporcionalidad (Montero y Riera, 2009: 428). 
arrancar ha sido un tibio compromiso para abordar la «mejora de la proporcionalidad» (medida 97), aunque el PP, justo en el párrafo siguiente, "se reserva la posibilidad de presentar iniciativas que permitan garantizar el Gobierno de la fuerza más votada», lo cual supone una contradicción flagrante con el compromiso anterior.

Una perspectiva de reforma muy diferente - en la línea de esta declaración de intenciones del PP que acabamos de citar- es la que plantearía la reforma, pero no para reforzar la proporcionalidad, sino para todo lo contrario. Como es sabido, en los sistemas parlamentarios de Gobierno los sistemas electorales están obligados a satisfacer simultáneamente dos objetivos que no siempre son conciliables entre sí: uno de ellos es conformar un Parlamento que sea representativo y el otro, desde la óptica del parlamentarismo racionalizado, es el de propiciar mayorías parlamentarias que puedan sustentar un Gobierno estable.

Hasta 2015, la dimensión de la gobernabilidad no había generado la más mínima preocupación a los estudiosos del sistema electoral. Es más, unánimemente se destacaba que nuestro sistema había posibilitado la formación de Gobiernos y propiciado su estabilidad en el tiempo (Martínez Pujalte, 2010: 48; Delgado-Iribarren, 2012: 6; López Nieto, 2016: 73). Sí, se admitía que nuestro sistema tenía problemas de desproporcionalidad y desigualdad, pero eran sacrificios asumibles en pos de la estabilidad gubernamental. Sin embargo, tras los últimos comicios la desproporcionalidad y la desigualdad no han desaparecido, pero, además, ahora se empieza a dudar de la eficacia del sistema para propiciar la formación de mayorías parlamentarias suficientes. A mi juicio, achacar alguna responsabilidad al sistema electoral sobre los problemas de gobernabilidad no responde en absoluto a la realidad. De hecho, como hemos visto, el sistema ha desplegado plenamente sus efectos de concentración de la representación.

¿Cabría alguna posibilidad de reforma que intentase reforzar aún más el efecto mayoritario del sistema? Desde luego no parece probable que se pudiera articular un consenso parlamentario en torno a una propuesta de esa naturaleza. El programa electoral y las declaraciones de líderes del PP parecen sugerir que a este partido le agradaría una solución de este tipo, mediante la articulación de un premio a la mayoría, pero no parece que ningún otro partido pueda sumarse a una propuesta asi ${ }^{17}$.

En estas circunstancias, todo parece indicar que las posibilidades de que se lleve a cabo una reforma electoral en esta legislatura son mínimas. Seguramente,

17 Para un análisis de posibles medidas de premio a la mayoría y de su posible implantación en España, véase Delgado-Iribarren (2012: 9-10). 
la reforma electoral seguirá estando presente en el debate público, para regocijo de expertos y tertulianos. Seguramente, el descontento ciudadano sobre el funcionamiento del sistema se incrementará. Pero, a pesar de ello, mucho me temo que, una vez más, se cumplirá la célebre aserción de Lijphart cuando afirmaba que los sistemas electorales "tienden a ser muy estables y resistentes al cambio" (1995: 52).

\section{UNA REFLEXIÓN FINAL: ¿̇SIMPLE REALINEAMIENTO O TRANSFORMACIÓN PROFUNDA DEL SISTEMA DE PARTIDOS?}

Para concluir este breve análisis, nos podemos plantear la cuestión de si, como consecuencia de los resultados electorales de 2015 y 2016, nos encontramos ante un simple realineamiento del sistema de partidos, de tal forma que el resultado final de este proceso podría volver a ser un sistema bipartidista imperfecto basado en la tradicional escisión ideológica izquierda-derecha, pero con otros actores distintos o, al menos, parcialmente distintos; en otras palabras, si podríamos estar dirigiéndonos hacia un nuevo bipartidismo, o hacia un multipartidismo bipolar, en el que Podemos asumiría el papel que ha asumido el PSOE hasta el momento como la fuerza política central del polo ideológico de la izquierda; o bien, si, por el contrario, estaríamos ante una transformación mucho más profunda del sistema de partidos, en el sentido de que ya no habría solo un cambio de actores, sino también un cambio en las líneas divisorias fundamentales (cleavages), con una pérdida de intensidad de la escisión ideológica tradicional izquierda-derecha y la irrupción de una nueva escisión que podríamos resumir en la oposición entre vieja política y nueva politica.

El análisis del comportamiento electoral en las elecciones generales de 2015 y 2016 (Rama Caamaño, 2016) nos permite observar que hubo síntomas de fatiga en el apoyo electoral de los partidos tradicionales y que los nuevos partidos (Podemos y Ciudadanos) fueron apoyados por electores muy distintos entre sí y que se alejan de la dimensión izquierda-derecha. Lo que tienen en común estos votantes es que, en términos generales, se muestran especialmente críticos con el funcionamiento de la democracia, con los partidos políticos y con «los políticos» (la casta, en la terminología puesta de moda en los últimos años). De esta forma, hay ciertos indicios de que podría estar cambiando la estructura de competición partidista y de que se estarían erosionando las viejas escisiones sociales (Rama Caamaño, 2016: 20).

Deteniéndonos específicamente en el caso de Podemos, parece claro que, en su etapa inicial, este partido responde claramente al perfil de partido antiestablishment. Sus apoyos en las elecciones europeas de 2014 procedieron de 
bolsas de votantes indignados, con fuertes sentimientos de rechazo a los partidos tradicionales y llenos de expectativas incumplidas (Rodríguez Teruel, 2016). En este sentido, Podemos se enmarcaba claramente en el fenómeno de auge de los movimientos populistas que se ha observado en diversos países, una «nube de populismo» (Solozabal, 2017) que, desde planteamientos ideológicos heterogéneos, coincide en «la descalificación del sistema político democrático, como tinglado institucional falso y esencialmente corrupto" (Solozabal, 2017), aunque destacando siempre la relativa excepcionalidad de Podemos, si lo comparamos con lo sucedido en otros países en los que el descontento ha sido capitalizado por movimientos asimilables de una u otra forma a la extrema derecha. Durante esa primera etapa fundacional, el éxito de Podemos se basó precisamente, como ha descrito con acierto Rodríguez Teruel (2016), en «un discurso populista hábilmente articulado, una gran capacidad para la agitación en las redes sociales y una notable presencia en grandes medios de comunicación». Estos rasgos iniciales permitían avalar la hipótesis de la sustitución de la dialéctica tradicional izquierda-derecha por la dialéctica vieja politica-nueva política. Sin embargo, la estrategia posterior seguida por Podemos, primero con las confluencias con los partidos nacionalistas y después con la alianza con Izquierda Unida, se aparta de esa línea inicial y vuelve a reforzar las líneas divisorias tradicionales del sistema español de partidos.

En definitiva, parece que Podemos habría optado claramente por el objetivo estratégico de reeditar bipartidismo, pero con otros actores, sustituyendo al PSOE como referente de la izquierda. El que este proceso de suplantación tenga éxito o no depende de una multiplicidad de factores cuyo análisis se escapa por completo a este modesto comentario. Uno de ellos será, sin duda, la resolución de las crisis abiertas en el momento actual tanto en el PSOE como en Podemos. Otro, de extraordinaria importancia, será la capacidad de las sociedades europeas para sobreponerse a la epidemia populista reafirmando los valores del constitucionalismo democrático.

\section{Bibliografía}

Delgado-Iribarren G.a-Campero, M. (2012). El sistema electoral español: logros y deficiencias. En Conferencia Los sistemas electorales español y alemán: Un debate de sus efectos, limites y propuestas de reforma (pp. 1-33). Fundación Manual Giménez Abad.

Duverger, M. (1957). Los partidos políticos. México: Fondo de Cultura Económica.

Fernández Esquer, C. (2015). Un sistema electoral que no garantiza la igualdad. Agenda Pública, 26-12-2015. Disponible en: http://agendapublica.es/un-sistema-electoral-queno-garantiza-la-igualdad/ 
— (2016). Desproporcionalidad y gobernabilidad. Agenda Pública, 29-3-2016. Disponible en: http://agendapublica.es/desproporcionalidad-y-gobernabilidad/

Fernández-Miranda Campoamor, A. (2009). Reflexiones sobre una improbable reforma del sistema electoral del Congreso de los Diputados. Revista de Derecho Político, 74, 19-46. Disponible en: https://doi.org/10.5944/rdp.74.2009.9067

García Viñuela, E. y Artés, J. (2009). Una estimación del voto estratégico en las Elecciones Generales Españolas, 2000-2008. Papeles de Trabajo del Instituto de Estudios Fiscales. Serie economía, 7, 3-29.

Garrote de Marcos, M. (2007). Las elecciones del 9 de marzo de 2008 y la igualdad de voto. Cuadernos de Derecho Público, 30, 53-78.

Lijphart, A. (1995). Sistemas electorales y sistemas de partidos. Madrid: CEC.

López Nieto, L. (2016). Notas sobre el rendimiento de los sistemas electorales en España (1976-2015). Revista Latinoamericana de Politica Comparada, 11, 69-107.

Maravall Herrero, J. M. y Santamaría Ossorio, J. (1989). Transición política y consolidación de la democracia en España. En J. F. Tezanos, R. Cotarelo y A. de Blas (eds.). La transición democrática española (pp. 183-249). Madrid: Sistema.

Martínez Pujalte, A. L. (2010). Los sistemas electorales españoles: evaluación y propuestas de reforma. Madrid: Dykinson.

Montero, J. R. y Riera, P. (2009). Anexo II: Informe sobre la reforma del sistema electoral. En F. Rubio Llorente y P. Biglino Campos (eds.). El Informe del Consejo de Estado sobre la reforma electoral. Texto del informe y debates académicos. Madrid: Centro de Estudios Políticos y Constitucionales.

Mora Molina, J. J. (2013). Calidad y democracia. Del sistema electoral a la rendición de cuentas. Madrid: Tecnos.

Moreno, C. y Oñate, P. (2004). Tamaño del distrito y voto estratégico en España. Reis, 107, 123-151. Disponible en: https://doi.org/10.2307/40184641

Nohlen, D. (2013). Controversias sobre sistemas electorales y sus efectos. Revista Española de Ciencia Politica, 31, 9-39.

Penadés, A. y Pavía Miralles, J. M. (2016). La reforma electoral perfecta. Madrid: Los Libros de la Catarata.

— y Santiuste Cué, S. (2013). La desigualdad en el sistema electoral español y el premio a la localización del voto. Revista Española de Ciencia Política, 32, 89-116.

Presno Linera, M. Á. (2015). Régimen electoral («maquiavélico») y sistema de partidos (con sesgo mayoritario). Revista Española de Derecho Constitucional, 104, 13-48. Disponible en: https://doi.org/10.18042/cepc/redc.104.01

Rae, D. W. (1977). Leyes electorales y sistemas de partidos politicos. Estudio comparativo. Madrid: Ediciones CITEP.

Rama Caamaño, J. (2016). Un nuevo terremoto electoral: elecciones generales 2015 y 2016 en España. Revista On-line de Estudiantes de Derecho (UAM), 5.

Rodríguez Teruel, J. (2016). Podemos: ¿De partido anticasta a estandarte de la plurinacionalidad? Agenda Pública, 12-12-2016. Disponible en: http://agendapublica.es/podemosde-partido-anticasta-a-estandarte-de-la-plurinacionalidad/

Rubio Llorente, F. (2014). Defectos de forma. Revista Española de Derecho Constitucional, $100,133-165$. 
Sánchez Muñoz, Ó. (1999). Sistema electoral y principio de igualdad de sufragio. En F. Pau i Vall (coord.). Parlamento y sistema electoral (pp. 491-528). Pamplona: Aranzadi.

- (2007). La igualdad de oportunidades en las competiciones electorales. Madrid: CEPC.

Sartori, G. (1994). Ingeniería constitucional comparada. México D. F.: Fondo de Cultura Económica.

- (1999). La ingeniería constitucional y sus límites. Teoría y Realidad Constitucional, 3, 79-87.

Solozabal Echavarria, J. J. (2017). La nube del populismo. El Imparcial, 13-1-2017. Disponible en: http://www.elimparcial.es/noticia/171424/opinion/la-nube-del-populismo.html

Soriano, R. y Alarcón, C. (2001). Las elecciones en España: ¿Votos iguales y libres? Revista de Estudios Políticos, 114, 115-130.

Torres del Moral, A. (2009). La reforma del sistema electoral o la cuadratura del círculo. Revista de Derecho Político, 74, 49-111. Disponible en: https://doi.org/10.5944/rdp. 74.2009 .9068

Urdánoz Ganuza, J. (2008). Ingeniería del sufragio: artimañas electorales vs. valores democráticos. Claves de la Razón Práctica, 180, 58-67.

- (2009). La desigualdad del voto en España. Anuario de la Facultad de Derecho de la Universidad Autónoma de Madrid, 13, 271-290.

Vázquez Lapuente, M. y Jiménez Seral, P. (2014). El nuevo modelo de reparto de escaños en el sistema electoral alemán. Cuadernos Manuel Giménez Abad, 7, 108-126.

Vidal Prado, C. (2012). El sistema electoral alemán como modelo: ventajas e inconvenientes. Asamblea, 26, 217-234. 